Measurement Notes

Note 63

March 2011

\title{
Automated and Adaptive RF Effects Testing
}

\author{
Everett G. Farr ${ }^{1}$, Leland. H. Bowen ${ }^{1}$, W. Scott. Bigelow ${ }^{1}$, Robert. L. Gardner ${ }^{2}$, \\ and Peter Finlay ${ }^{3}$ \\ ${ }^{1}$ Farr Fields, LC, Albuquerque, NM \\ ${ }^{2}$ Consultant, Alexandria, VA \\ ${ }^{3}$ Air Force Research Laboratory, KAFB, NM
}

\begin{abstract}
Testing electronics for vulnerability to radio frequency (RF) radiation is time-consuming, due to the large number of source variables of interest. One typically searches for the minimum electric field that causes upset, as a function of center frequency, pulse width, pulse repetition frequency, number of pulses, and bandwidth. It is impossible to test all combinations of all the variables, so one must intelligently select the source parameters most likely to expose the greatest vulnerability. To select source parameters, we propose using standard techniques from minimization theory. Within a space of two or more variables, we search for the combination that upsets the system at the lowest power or field level. We investigated the vulnerability of media converters (MCs) to pulsed RF fields. We tested these devices by pinging a remote computer, and observing the field levels at which the pings failed to return.
\end{abstract}




\section{Introduction}

The vulnerability of electronics to radio frequency (RF) fields has been well documented, for example in $[1,2]$. This has led to a major effort to test electronics to find the minimum field or power at which an effect is observed. However, such testing is time-consuming, due to the large number of source variables of interest. One typically searches for the minimum electric field that causes upset, as a function of center frequency, pulse width, pulse repetition frequency, number of pulses, and bandwidth. It is impossible to test all combinations of all the variables, so one must intelligently select the source parameters most likely to expose the greatest vulnerability.

To select source parameters, we propose using standard techniques from minimization theory. Within a space of two or more variables, we search for the combination that upsets the system at the lowest power or field level. We begin by measuring the vulnerability levels on a coarse grid; and then fit a surface to the measured data. We then find the minimum of the surface, and measure the vulnerability at the minimum. With the new data, the process repeats iteratively until it converges.

Ideally, the entire process can be automated. The source variables can all be controlled electronically. In addition, one can determine automatically whether the test object has been upset, and send a reset command if necessary. This leads to a completely automated system that intelligently selects the test parameters, monitors the status of the device, and converges on a minimum upset threshold. During this first implementation, some manual operations were required; however, these can be automated at a later date.

In this project, we investigated the vulnerability of media converters (MCs) to pulsed RF fields. MCs are network devices that convert signal on Cat 5 Ethernet cable to optical fiber, and are known to be vulnerable. We tested these devices by pinging a remote computer, and observing the field levels at which the pings failed to return. We searched a space of source variables, and converged on a minimum upset threshold. Most of the operations were carried out automatically. 


\section{Experimental Setup}

After much consideration, we decided to test the vulnerability of media converters to RF effects. These devices convert data between Cat 5 Ethernet cables and fiber optic cables. Such devices are commonly used in networks, and are known to be vulnerable.

Media converters are particularly easy to configure for vulnerability testing. They are small, and fit into a standard TEM cell that is commonly used to test cell phones. They degrade gracefully when stressed by RF, causing a delay in network traffic. This delay can be quantified in a simple 2-computer network, in which one computer pings the other, and records the time delay between outgoing and returning messages.

The MC we tested was the IMC model TP-TX/FX-MM850-ST, operating at $850 \mathrm{~nm}$. This was selected because of its low cost and easy availability. A photo of this MC is shown in Figure 1. When configured for testing, the MC requires connections for two optical cables, an Ethernet cable, and a power cable. The two optical cables are necessary in order to communicate in both directions.

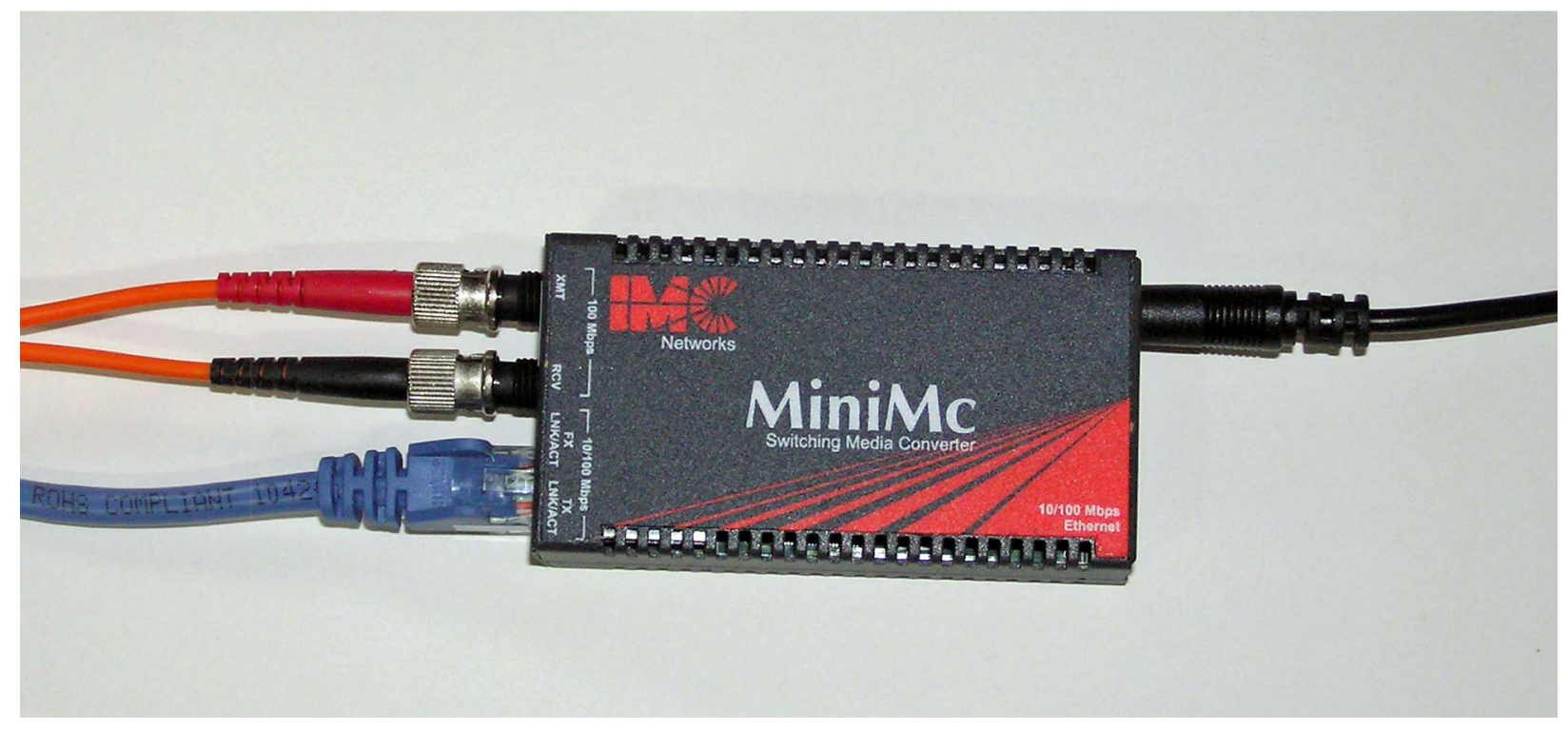

Figure 1. The IMC model TP-TX/FX-MM850-ST media converter, showing two optical cables (upper left), the Cat 5 Ethernet cable (lower left), and the power cable (upper right).

We tested the device in a CTS model 5062A TEM cell, a photo of which is shown in Figure 2. A typical experimental setup is shown in Figure 3 (left), and a field pattern is shown in Figure 3 (right). 


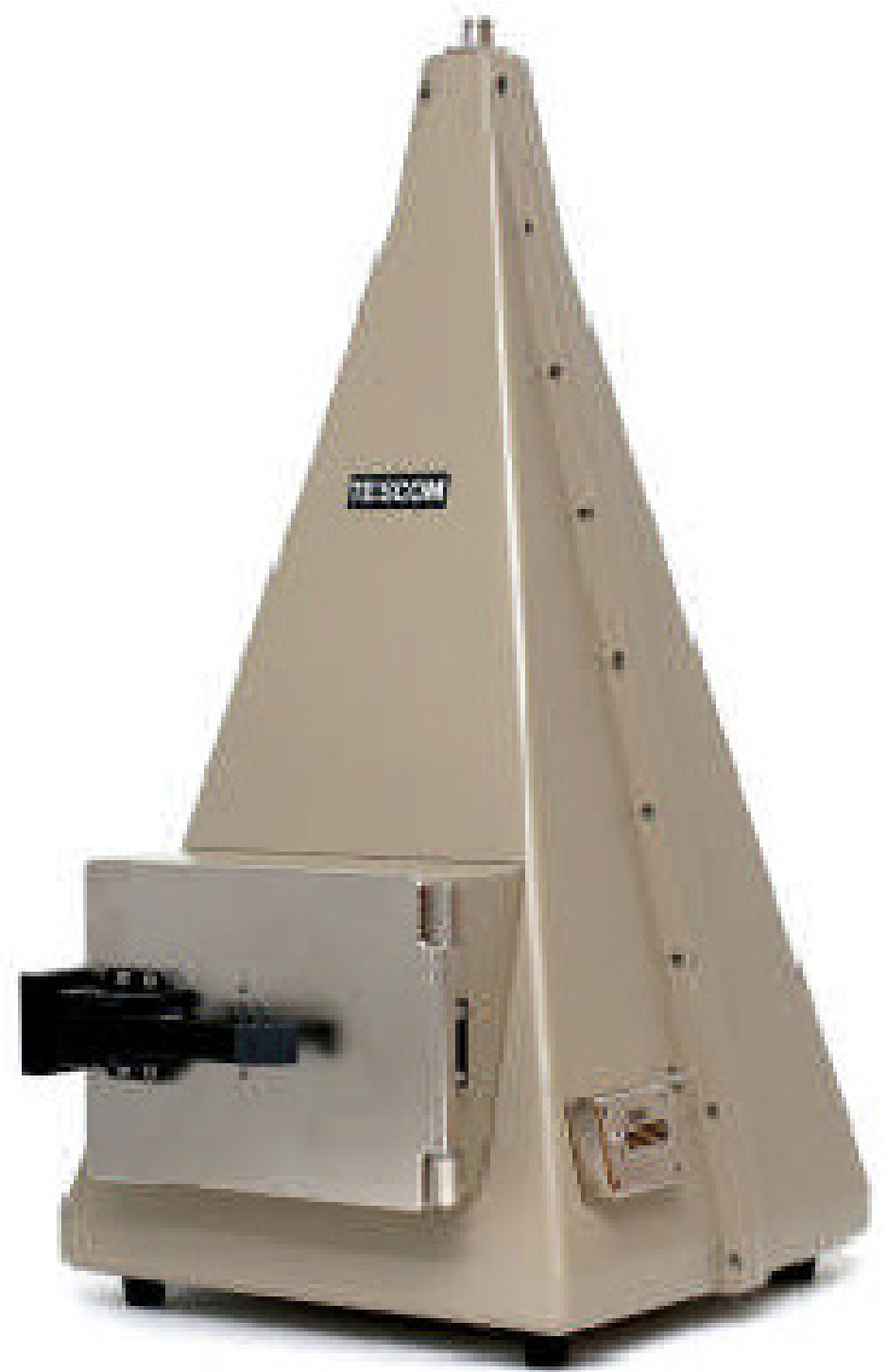

Figure 2. Photo of the CTS model 5062A TEM Cell. 

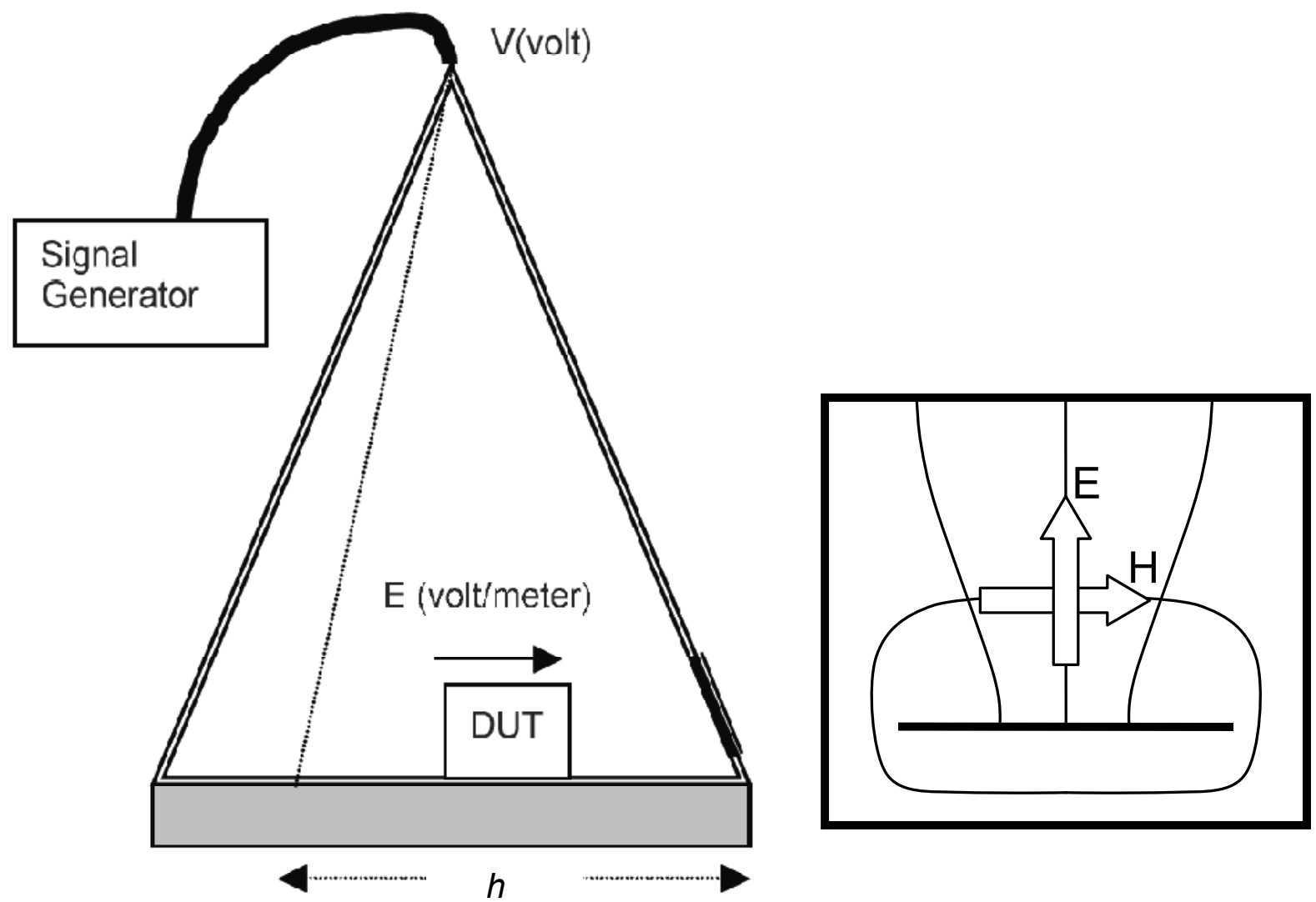

Figure 3 Typical setup of the CTS model 5062A TEM Cell (left) and approximate field pattern (right). 
We tested the MCs in the configuration shown in Figures 4 and 5. The main computer, running LabVIEW code, pings a remote computer through four MCs, two lengths of fiber optic cable, and three lengths of Cat 5 network cable. The computer controls the parameters of the synthesizer, which drives the amplifier that feeds into the TEM cell. Software running on the main computer then pings the remote computer, listens for the return signal, and detects a failure to respond.

This configuration, which tests two MCs concurrently, was chosen in order to limit RF leakage from the TEM cell. The penetrations of the TEM cell were either fiber optic cables or filtered DC power cables, both of which could be configured to maintain the integrity of the RF shield. Previously, we tested a configuration with a single MC in the TEM cell; however, the metallic Cat 5 cable that penetrated the wall of the TEM cell caused excessive RF leakage. Attempts to limit this leakage by filtering the Cat 5 cable at the wall of the TEM cell resulted in loss of network signal.

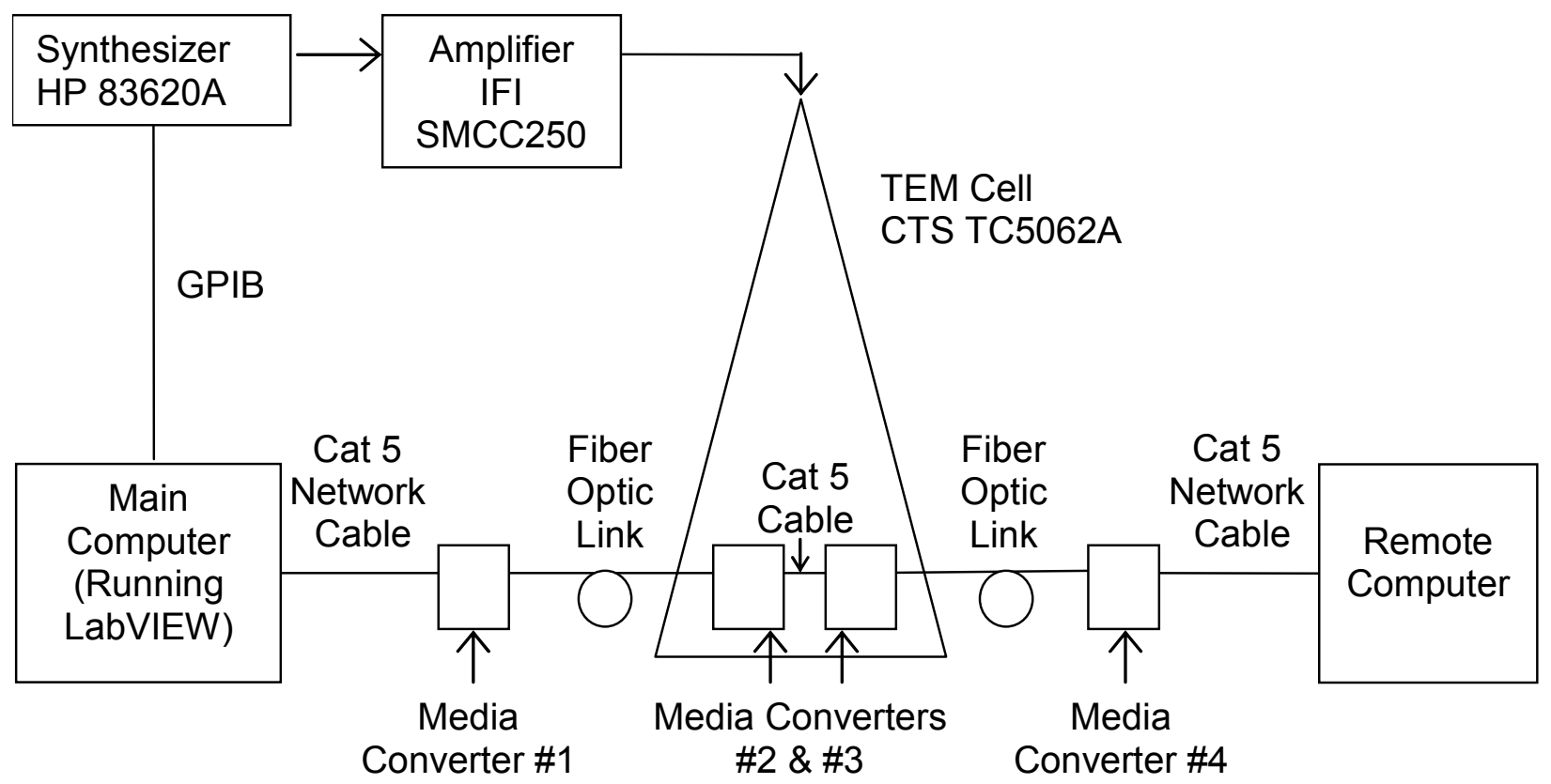

Figure 4. Experimental setup to test degradation of MCs with exposure to RF. 


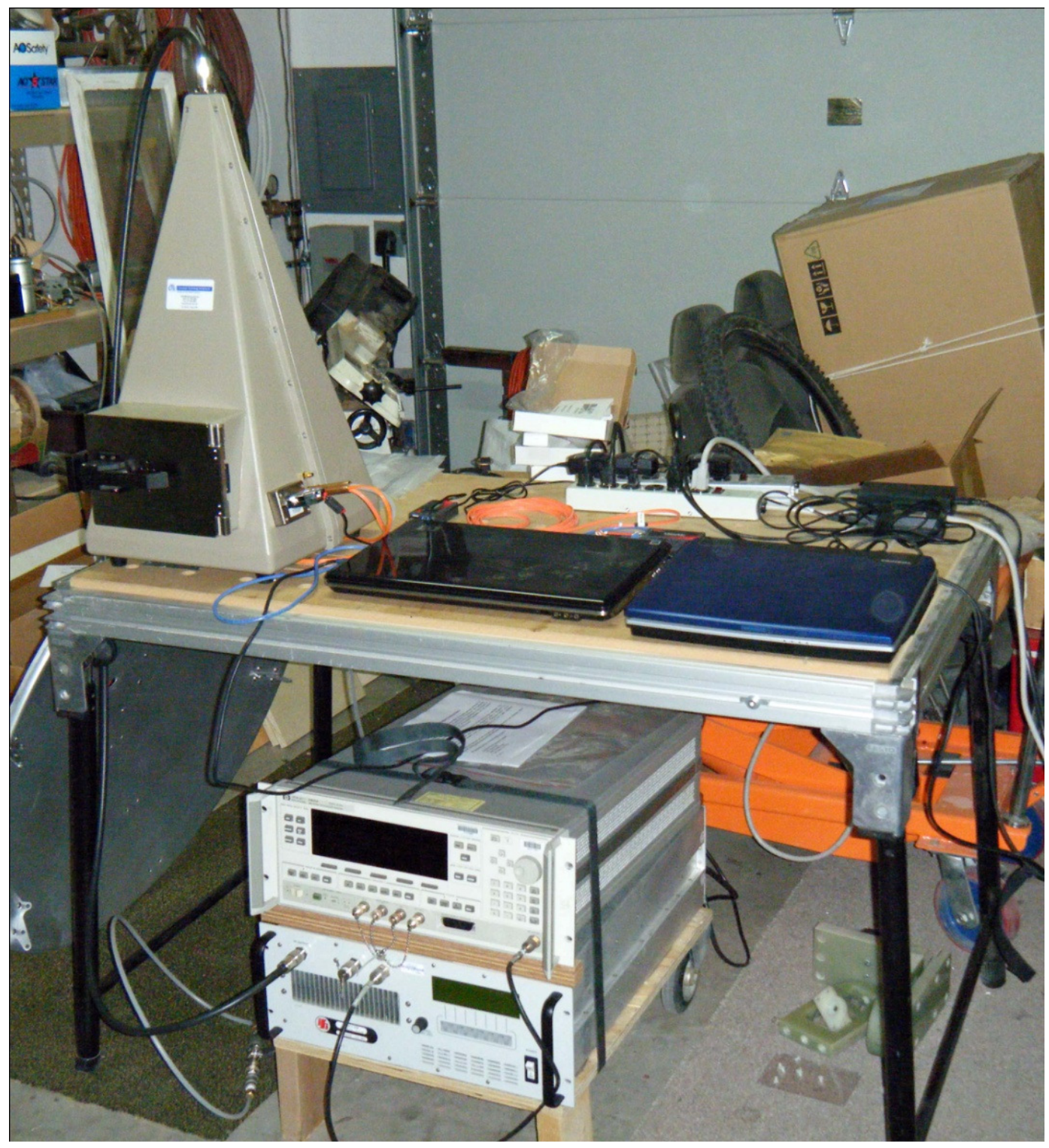

Figure 5. Photo of the experimental setup. 
The TEM cell was penetrated in two places, as shown in Figure 6. The first penetration was a DB- 25 connector, filtered by a $100-\mathrm{pF}$ capacitance. This connector provided DC power to the MC. The second penetration was a waveguide beyond cutoff (WBC), which allowed the penetration of optical cables. The WBC had an inner diameter of $1 / 2$ " and length of $3 "$. We estimated the cutoff frequency for this circular cross section waveguide to be $18 \mathrm{GHz}$.

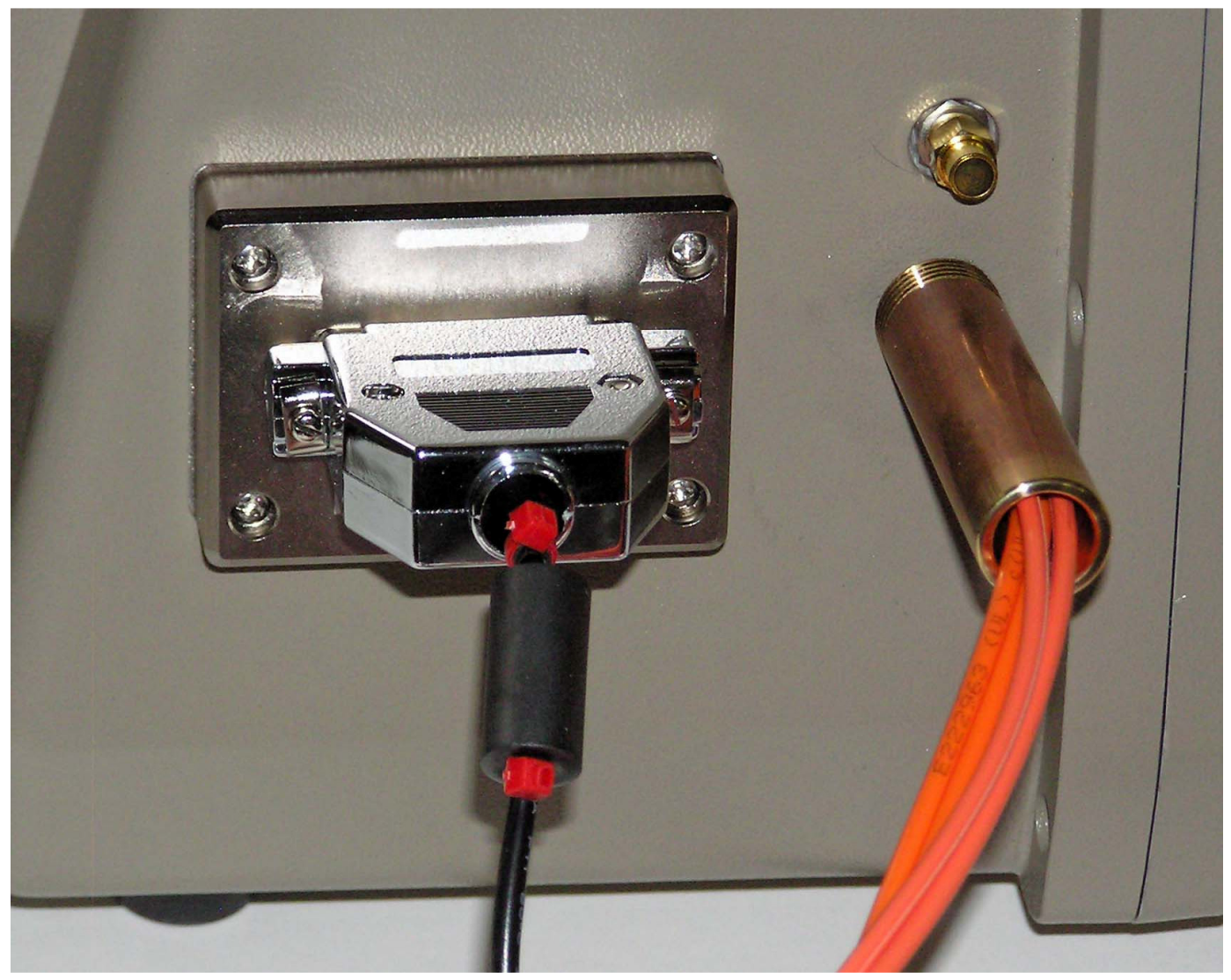

Figure 6. Two penetrations of the TEM cell: the filtered DB-25 connector for power transmission, left, and the waveguide beyond cutoff for optical fiber penetration, right. 
The interior of the TEM cell is shown in Figure 7. Note that the interior is somewhat cluttered with cables. Changing their exact position might affect the outcome of the measurements.

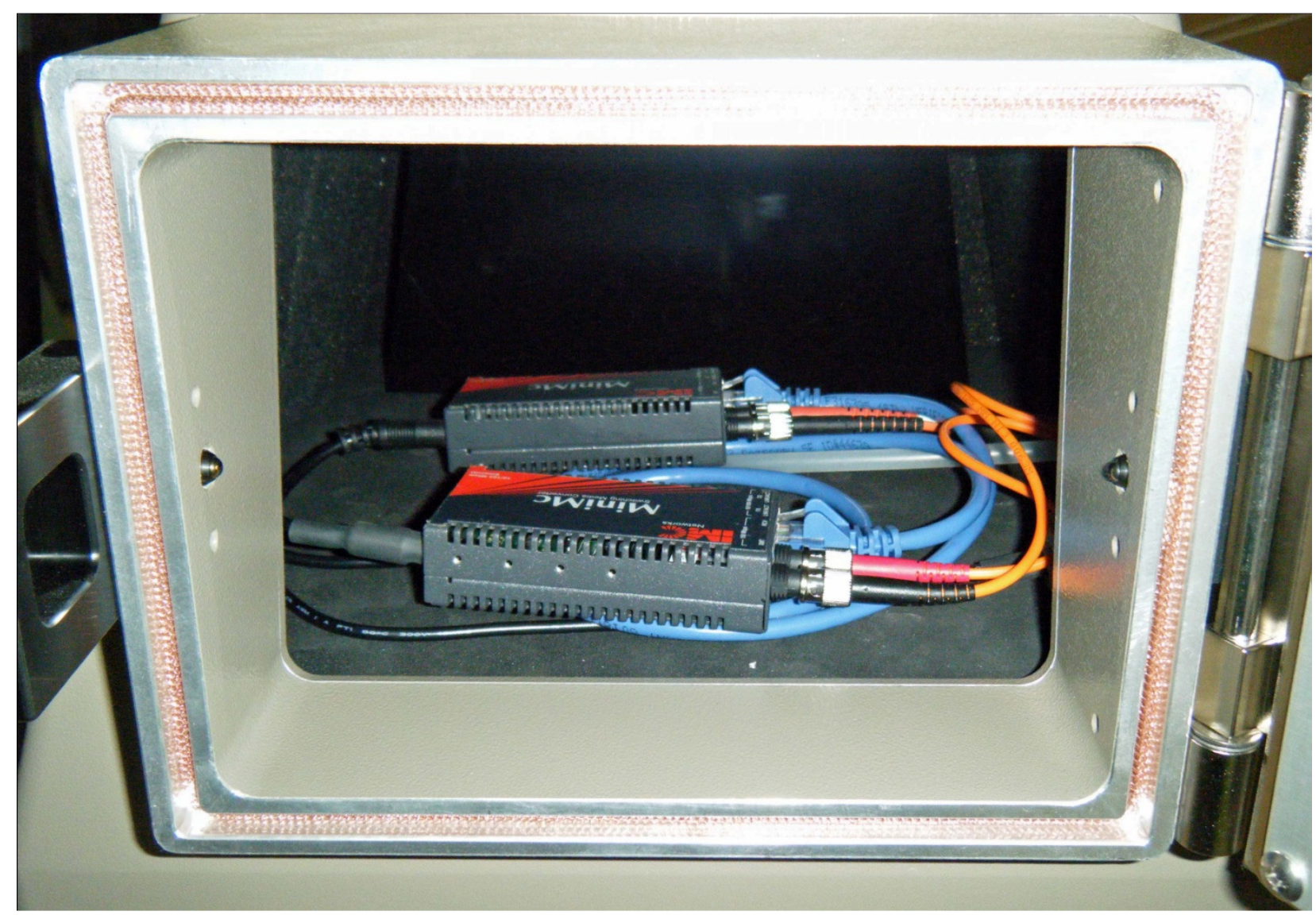

Figure 7. The interior of the TEM cell, showing two MCs, a blue Cat 5 cable between them, and orange fiber optic cables that penetrate the shield.

It is necessary to relate the power out of the amplifier to the field in the TEM cell. To do so, we observe that power at the input is converted to voltage as

$$
P_{p}=\frac{V_{p}^{2}}{2 \times 50 \Omega}, \quad V_{p}=\sqrt{P_{p} \times 100 \Omega}
$$

where $P_{p}$ is the peak power, and $V_{p}$ is the peak voltage in the sine wave on the $50-\Omega$ feed line. Note that power in this paper always refers to power averaged over at least one cycle of a sine wave. When we modulate the input signal with a square wave, peak power is the power (averaged over a sine wave) when the signal is turned on, and average power is averaged over on cycle of the square wave. 
The peak field in the TEM cell is approximately related to the voltage at the input as

$$
E_{p}=\frac{V_{p}}{h}, \quad h=0.22 \mathrm{~m}
$$

where $h$ is the plate separation in the test volume, as shown earlier in Figure 3 . This is an approximate average value suggested by the manufacturer. This approximation is not valid near the edges of the center conductor, where the fields are very high. Finally, the average power is

$$
P_{a v g}=P_{p} \times D F, \quad D F=P W \times P R F
$$

where $D F$ is the duty factor, $P W$ is the pulse width, and $P R F$ is the pulse repetition frequency. In this case, the duty factor is the fraction of time the square pulse of $\mathrm{CW}$ energy is turned on. 


\section{Software}

The software consists of two pieces of code, a threshold detector and a minimization routine.

The threshold detector determines automatically the minimum field level required for upset for a given set of source parameters. It sets the frequency, pulse width (PW), and pulse repetition frequency (PRF) of the synthesizer. The power level is initially set to a low value, and is gradually incremented. At each increment, the remote computer is pinged 20 times. When the power is high enough to yield 3 failures out of 20 pings, it is considered an upset condition, and that level is the upset threshold. This entire procedure is carried out in code that was written in LabVIEW. A screen shot of the threshold detector is shown in Figure 8.

The minimization routine guides the selection of parameters to test, in order to iterate to find a minimum upset threshold. We choose two variables over which to search, pulse width, and either duty factor or frequency. The procedure begins by taking data at nine points in the data space, the minimum, center, and maximum of each variable. A surface is fitted to this initial set of data using the "fminsearch" function in MATLAB, which then finds the minimum of the surface. This minimum is then used as the next point to test. The new results are added to the previous data, a new surface is fitted to the data, and a new minimum is found. The process repeats until the result converges. A screen shot of the minimization routine is shown in Figure 9.

To fully automate the process, it will be necessary to have the two pieces of code talking to each other. Thus, the threshold detector, written in LabVIEW, will have to talk to the minimization routine, which is written in MATLAB. This might sound challenging to do, but we are currently doing something similar in our antenna measurement software. So it will be straightforward to implement this during Phase II. 


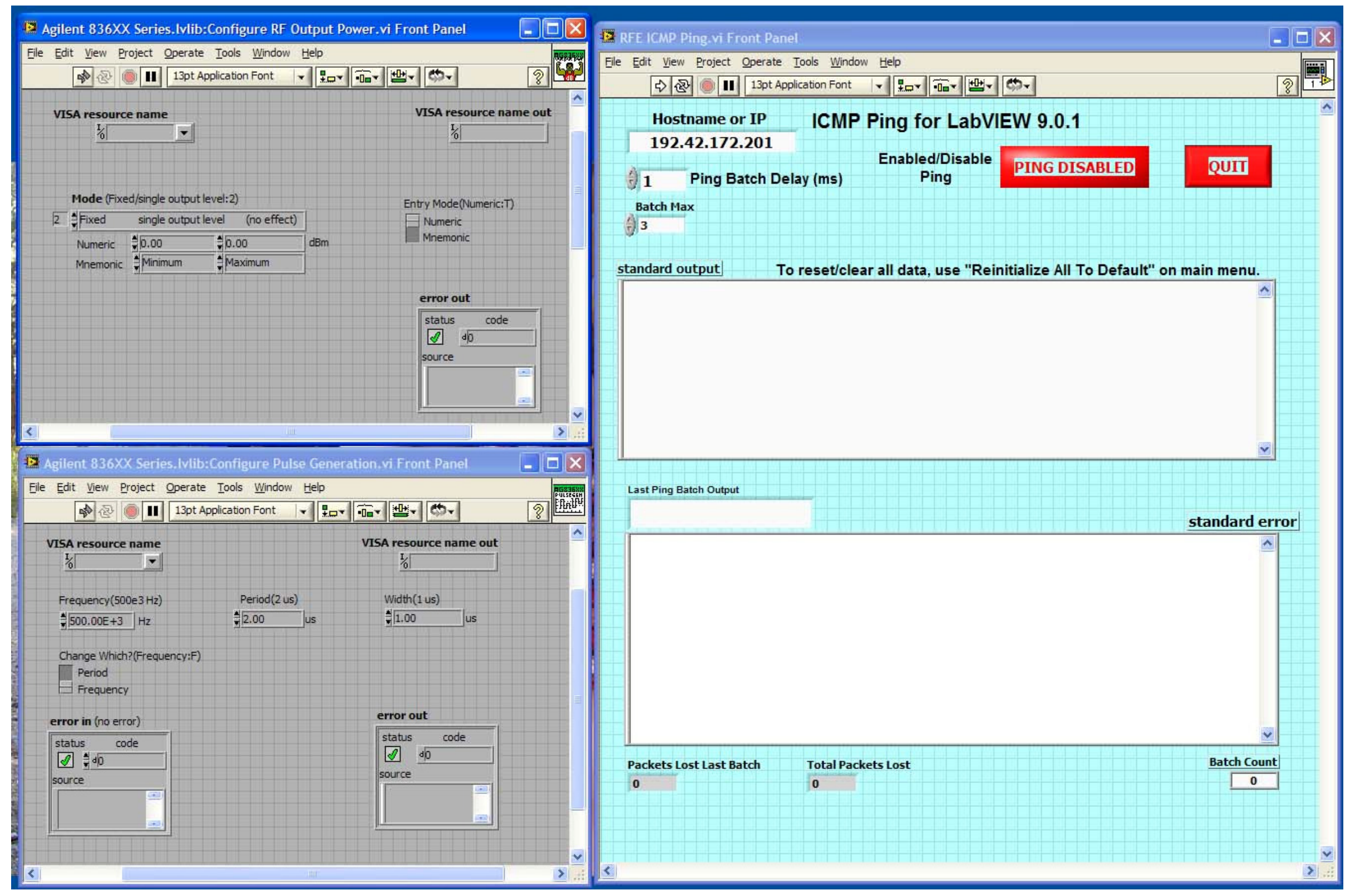

Figure 8. Front panel of the threshold detector software. 


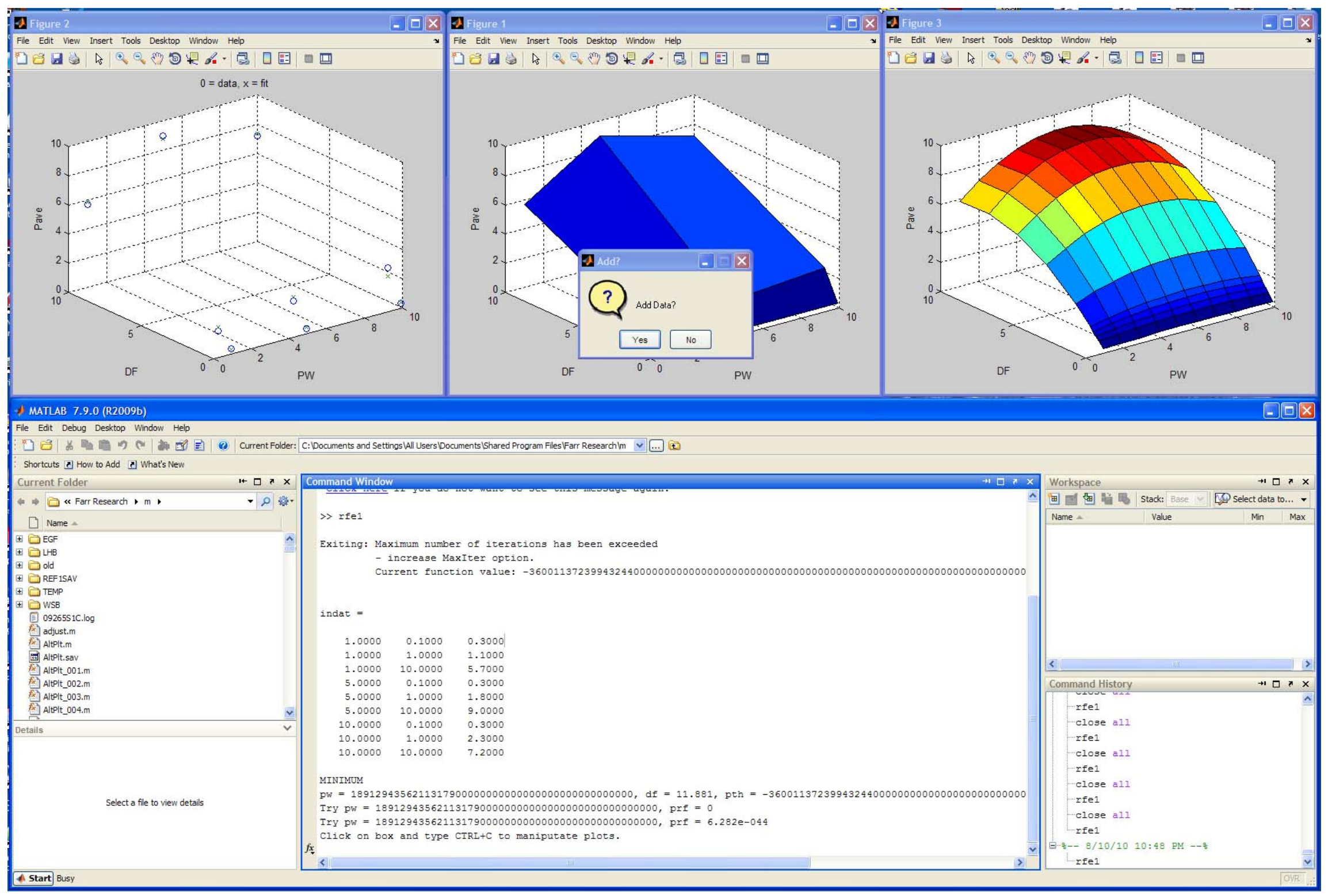

Figure 9 Front panel of the minimization software. 


\section{Data Showing Convergence in the Middle of the Test Space}

A key goal was to locate a minimum in the middle of a vulnerability test space. In a number of early experiments we found minima at less interesting locations-either at a corner or edge of the test space. However, finding a minimum in the middle of the test space demonstrates the usefulness of our minimization algorithm. We do so here.

We tested the vulnerability of two media converters in our TEM cell, using the configuration shown earlier in Figure 4. We tested with a pulse width $(P W)$ of $10 \mu \mathrm{s}$, with frequency $(f)$ ranging over $700-900 \mathrm{MHz}$, and duty factor $(D F)$ ranging over $0.1-10 \%$. The original nine points in the space are shown in Figures 10 and 11, with the raw data on the top and the surface fit on the bottom. Data are plotted in terms of peak power units. We have left the power units arbitrary intentionally. Plots on a linear scale are in Figure 10, and the same plots on a semi-log scale are in Figure 11.

We then iterated three more times to find the minimum, and the result is shown in Figure 12. The minimum converges to a frequency $(f)$ of $805.1 \mathrm{MHz}$, and duty factor $(D F)$ of $7.8 \%$, where we found a peak power $\left(P_{p}\right)$ of 0.010 , based on the curve fit. We then measured a point very close to the minimum, at $f=800 \mathrm{MHz}$ and $\mathrm{DF}=8 \%$, where we found $P_{p}=0.018$.

Thus, we observed $P_{p}=0.010$ in the curve fit, and we measured $P_{p}=0.018$ very nearby. This is a little less accurate than we would like to see, but it is still very good.

We should be able to improve the fit of the surface to the data by giving greater weight to data that is closer to the minimum. This is appropriate, since it is that portion of the surface in which we are most interested. We can do so, for example, by fitting to the inverse square or inverse cube of the vulnerability function. We hope to do so in future work. 

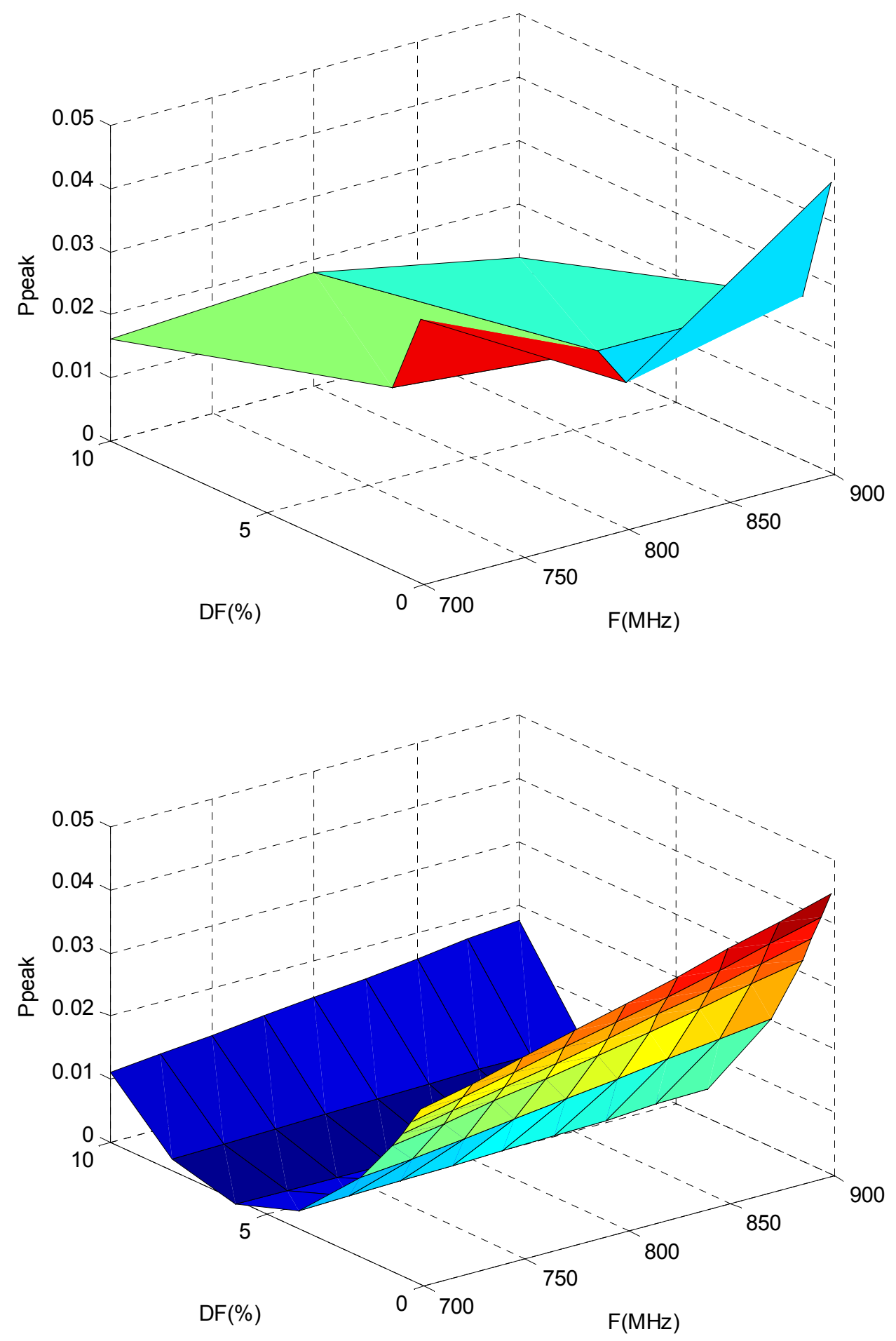

Figure 10. The original 9 points in the MC vulnerability test, top, and surface fit, bottom, on linear scales. 

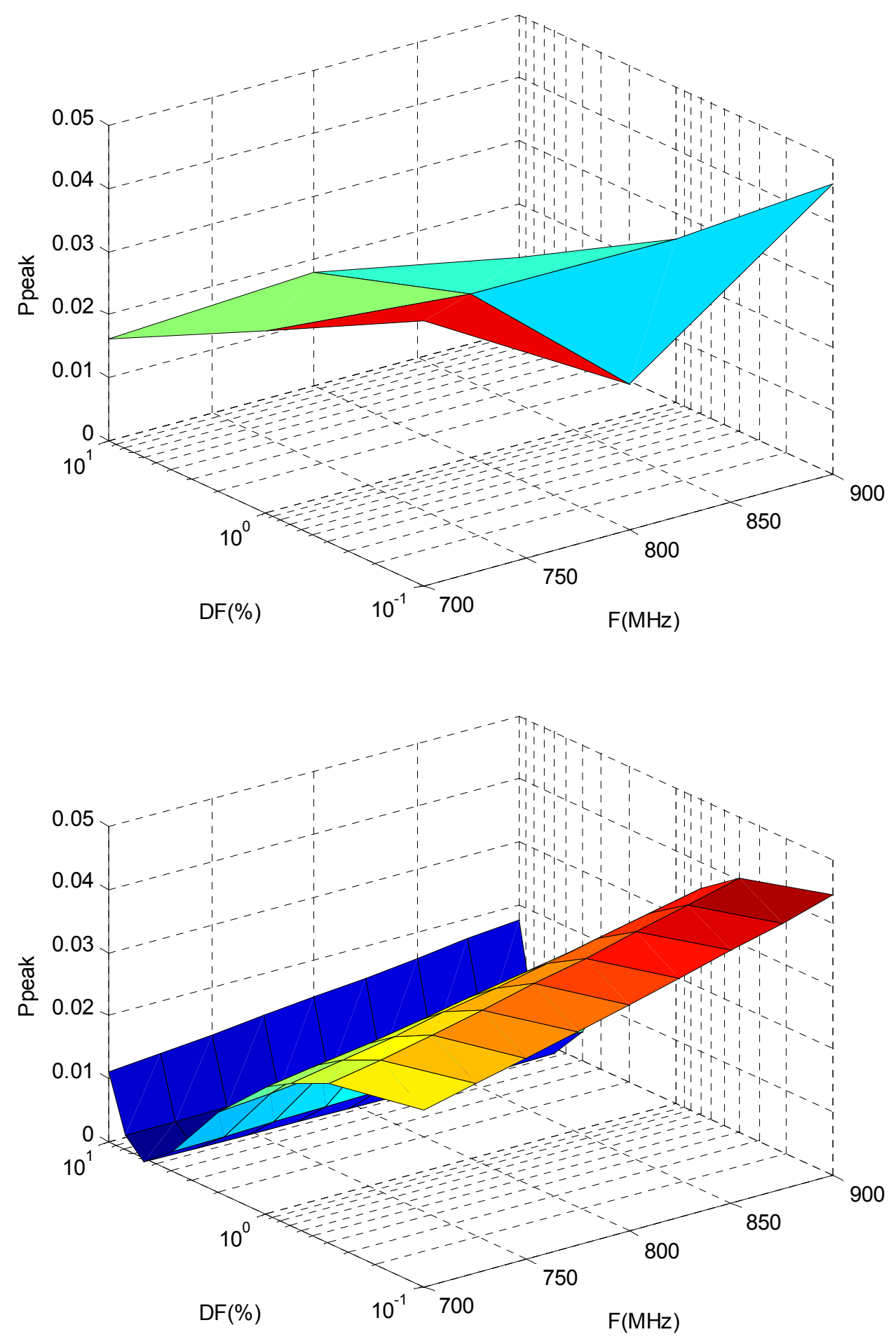

Figure 11. The original 9 points in the MC vulnerability test, top, and surface fit, bottom, on semi-log scales. 

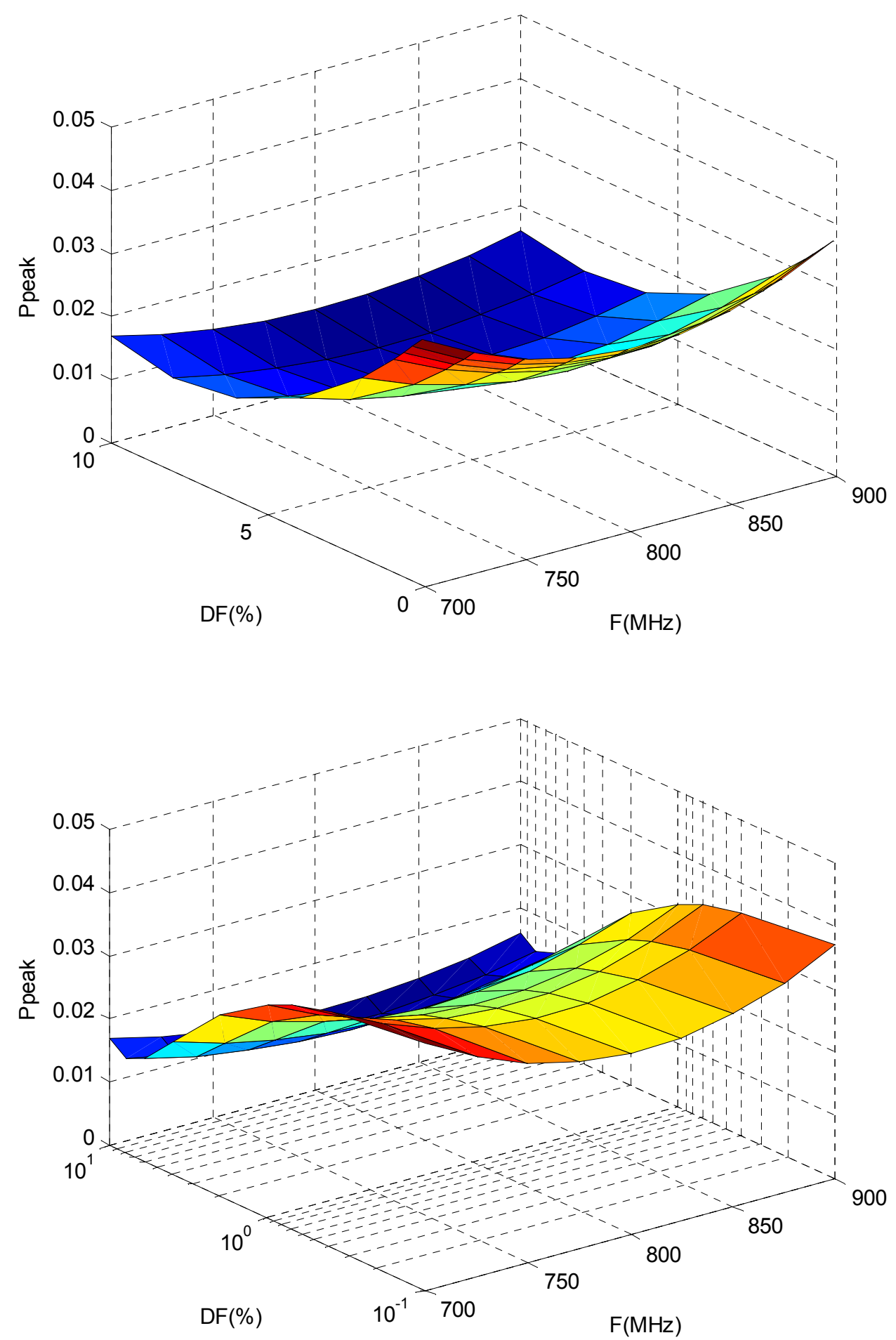

Figure 12. Surface fits of the data after adding 3 more points close to the minimum; linear scale on the top, and semi-log scale on the bottom. 


\section{Reproducibility of Results}

We investigated two types of reproducibility in upset thresholds:

1) The test setup is undisturbed from one experiment to the next.

2) The DUTs are removed and re-installed. In this case, cables are no longer in exactly the same position, so one would expect greater variability in the results.

Three measurements were made to test the two types of reproducibility - an initial test, a second test in which the configuration was undisturbed, and a third test after removing and reinstalling the DUTs. Upset thresholds are shown in Figure 13, where we see that as long as the configuration is undisturbed, the data are highly reproducible. However, if the system is disturbed, there is some variability in the measurements.

In any practical test, the configuration of cables will make a significant difference. It will be of interest in the future to investigate cables oriented parallel and perpendicular to the electric field. More often, however, cables are coiled, or just tossed in some random position. All of these cases will have to be studied in order to fully understand the problem. 

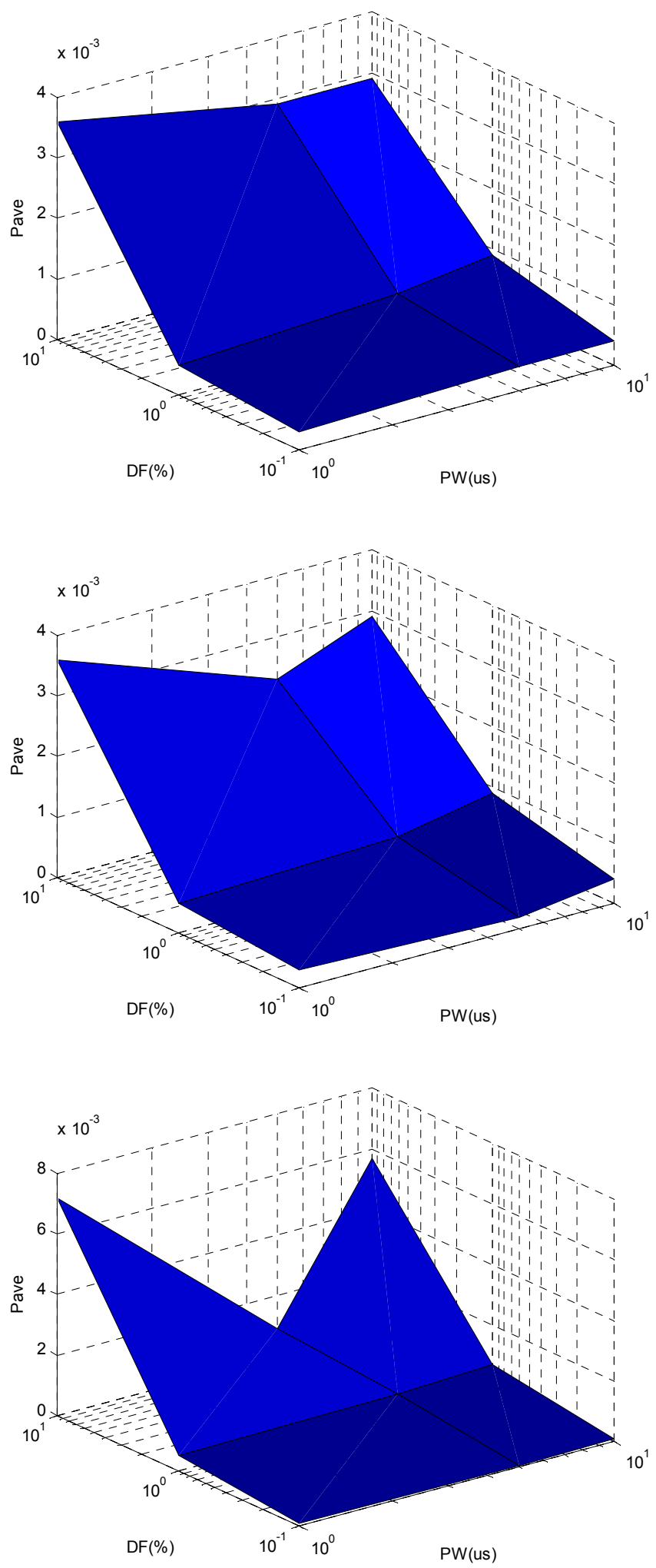

Figure 13. Reproducibility of upset thresholds - initial test (top), second undisturbed test (middle), and test after disturbing the configuration (bottom). 


\section{Cable Current Measurements}

One way to identify the frequency with the lowest upset threshold might be to select the frequency that has the highest current on the Cat 5 cable. To see that maximum, we connected a network analyzer between the input of the TEM cell and a current sensor, and measured $\mathrm{S}_{21}$. We investigated cases for which the cable is mostly perpendicular and mostly parallel to the electric field, and the results are shown in Figure 14. We observe that the detected current is much more flat than we expected, without sharp resonances. We also observe that the case with the cable mostly parallel to the field has a higher current, as one would expect. 

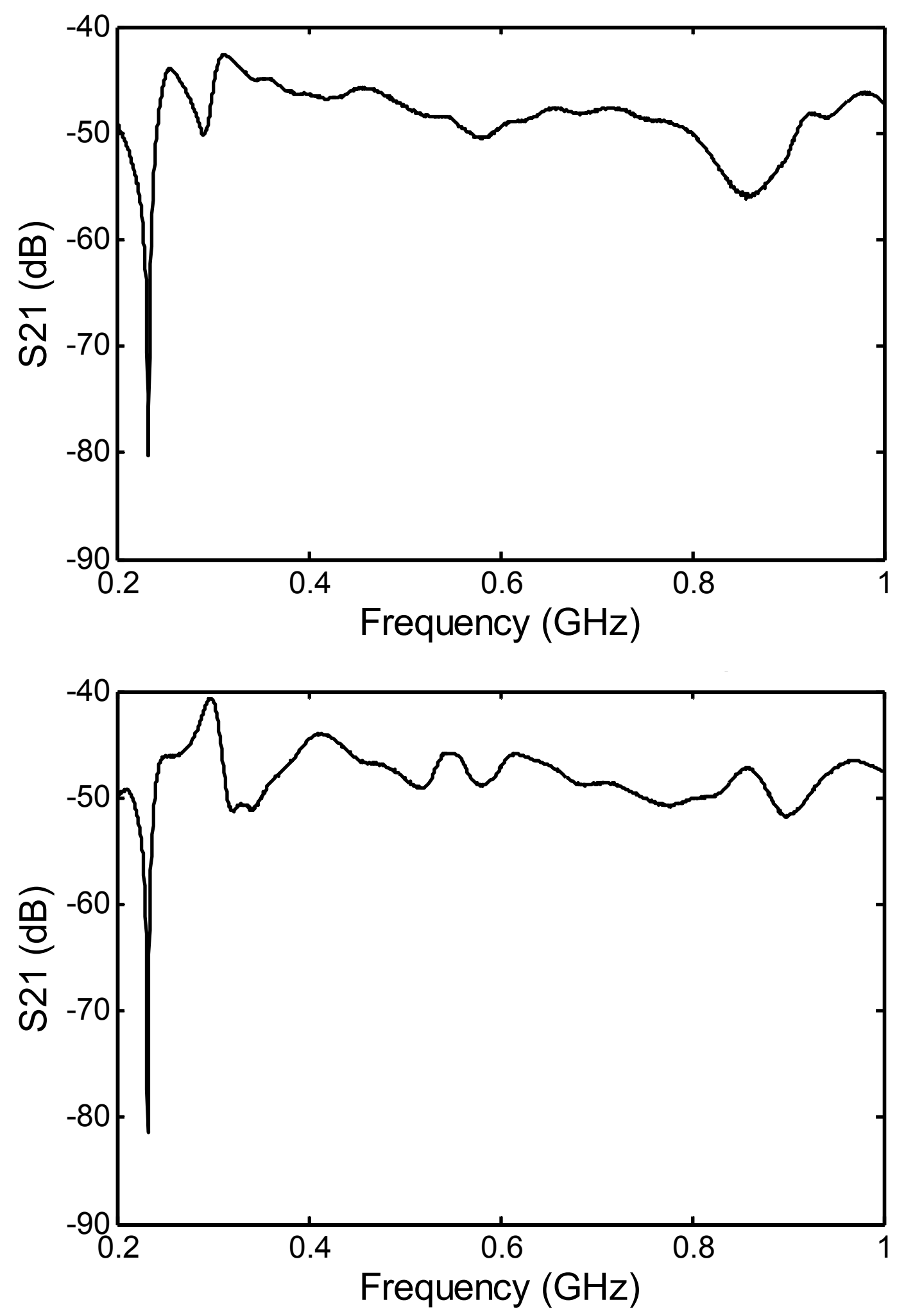

Figure 14. Current measurement on Cat 5 cable, with cable oriented mostly perpendicular (top) and mostly parallel (bottom) to the electric field. 


\section{Short Cable vs. Long Cable}

Next, we tested the effect of cable length on the upset threshold. The two MCs are connected in the test chamber with a Cat 5 cable as shown in Figure 15.

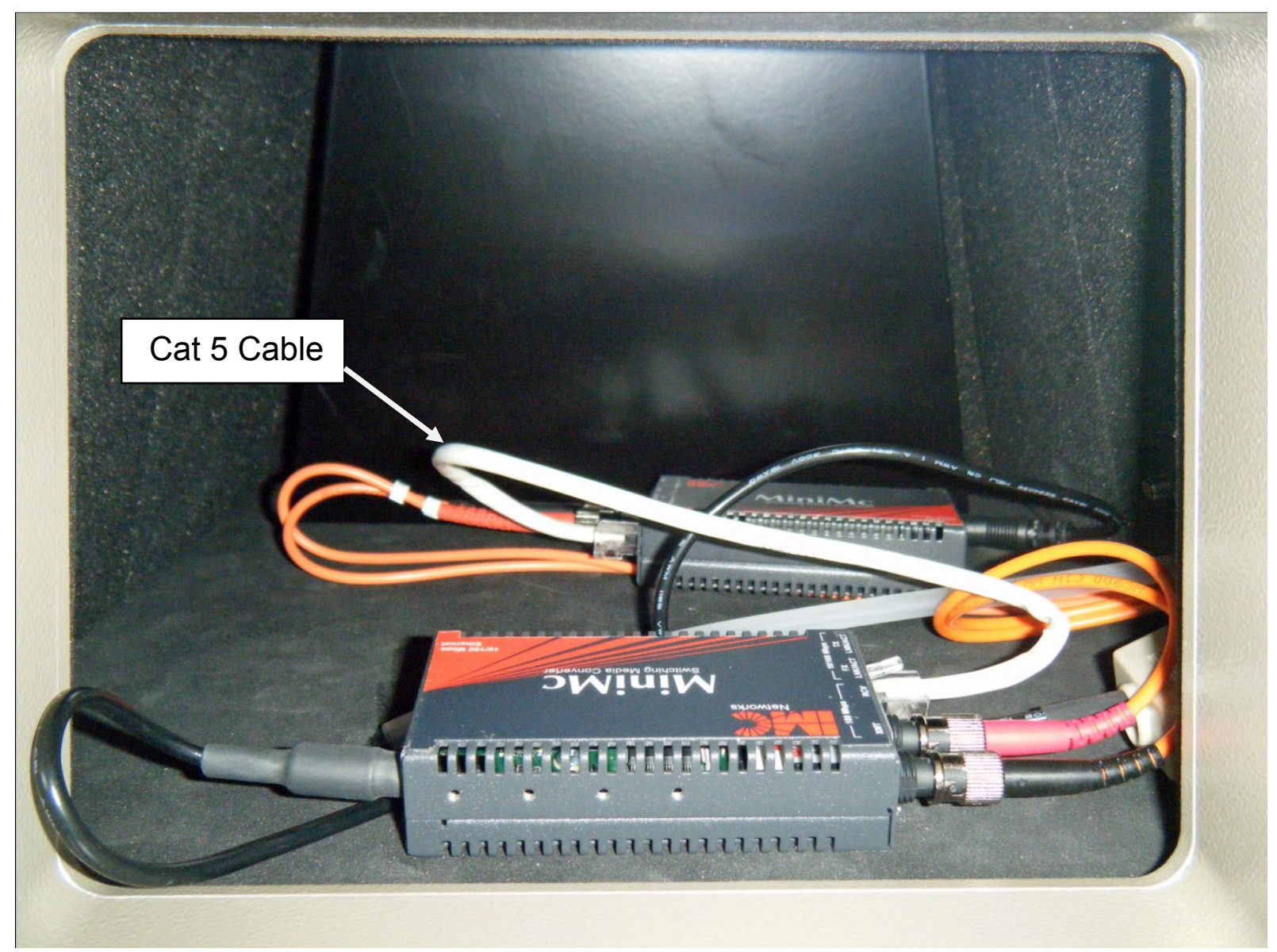

Figure 15. Interior of TEM cell, showing two MCs connected by a Cat 5 cable.

If the cable were the dominant coupling path, then one would expect lower upset thresholds with longer cables. We tested two lengths of Cat 5 cable-12 in. and 36 in. Indeed, the configuration with the longer cable is slightly more vulnerable, as shown in Figure 16. 

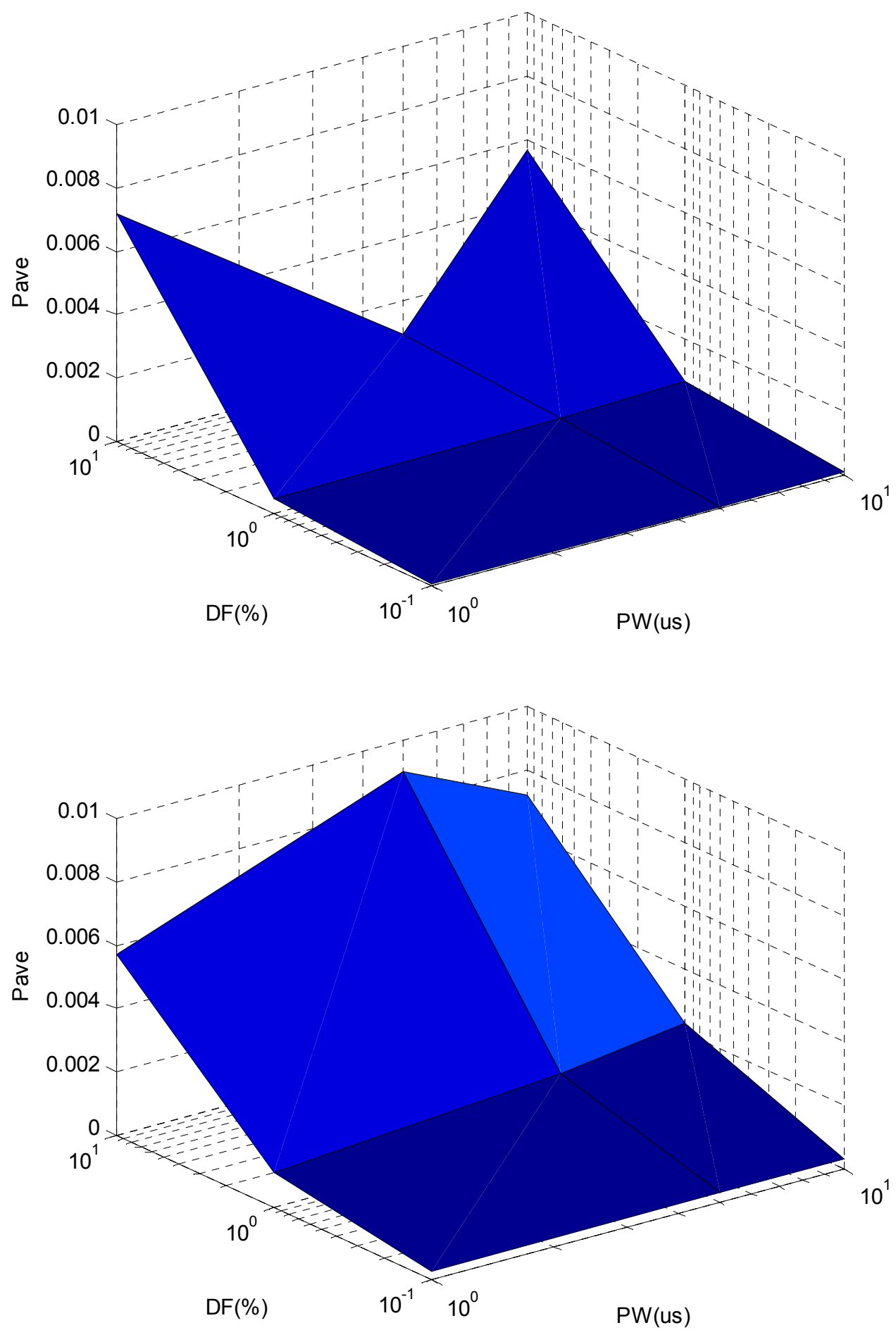

Figure 16. Upset thresholds with 36-in cable (top) and 12-in. cable (bottom). 


\section{Future Work}

We outline here a number of areas that would benefit from further work.

First, it would be necessary to fully integrate the software into a single unit. Currently, the software exists in two separate programs, which leads to manual operations. These programs have to be integrated in order to realize a fully automated system.

Second, we would have to add an automatic power characterization to each measurement, using a directional coupler and oscilloscope. This would involve making the software talk to the oscilloscope, downloading the voltage waveform, and converting the measured voltage to peak power. Ideally, one would prefer having an amplifier whose power is described by its dial settings, however, that seems to be difficult to realize in practice.

Third, we would investigate a number of variations on our minimization algorithm. For example, we would investigate alternative surface functions to fit to our data. In this paper, we used a function of the form

$$
z=\left(a x^{2}+b x+c\right)\left(d y^{2}+e y+f\right)
$$

where $x$ and $y$ are the two variables over which we are minimizing, and $a, f$ are the unknown coefficients that are chosen to give the best-fit surface to the measured data. However, many other functional forms are possible. For example, one could use higher order polynomials or spline functions.

Fourth we would investigate methods of giving greater weight to the function value near its minimum. The current method simply implements a least-mean-square fit to the measured data. However, the data close to the minima are of greater interest, so it is more important to reduce the fitting error in that region. To emphasize the minima, one might fit a surface not to the data itself, but to its inverse square or inverse cube. By this method, errors near the minima carry more weight, and therefore are reduced in the fitted function.

Fifth, note that in this project we searched a two-dimensional space for the minimum upset threshold. However, this technique should apply equally well to searches in higher order spaces, and this should be examined.

Finally, we would test a variety of other devices, which might include cell phones, iPods, and/or network routers. The idea here would be to incorporate alternative upset modes and reset mechanisms into the programming. One could detect an upset by listening (electronically) for the music on a telephone or iPod to stop. One could also detect when a screen goes dark with a photodetector. One could reboot a system after upset by electronically toggling a power switch. One could use a servomotor to twist a knob on a source. 


\section{Conclusions}

We have automated the testing of media converters for vulnerability to RF effects. Our testing involved pinging a remote computer, and listening electronically for missing return signals. To do this, we used software written in LabVIEW and MATLAB.

The most important result is that we have successfully observed a minimum in the middle of a test space. This is the first nontrivial use of the minimization algorithm, so it is a significant milestone.

This is the first step of what we imagine will be a long journey. A list of the future work that we envision is provided in Section VIII of this report.

\section{References}

1. D. Nitsch, F. Sabath, H.-U. Schmidt, and C. Braun, Comparison of the HPM and UWB Susceptibility of Modern Microprocessor Boards, System Design and Assessment Note 36, July 2002.

2. M. Camp, D. Nitsch, F. Sabath, J.-L. ter Haseborg, H. Garbe, Susceptibility of Some Electronic Equipment to HPEM Threats, System Design and Assessment Note 37, February, 2004. 